the existence of different condensed states.

The most common assay of gelation, minimal gelling concentration, depends in part on kinetic factors. A better measure of thermodynamic properties is obtained from the recently developed $C_{\text {sat }}$ assay in which a preformed gel is sedimented and the concentration of the supernatant, consisting of monomers previously in equilibrium with the condensate, is obtained. $C_{\text {sat }}$ is independent of total haemoglobin concentration and is thus a saturating concentration which reflects a phase separation $^{8-11}$. Magdoff-Fairchild et al. suggest the possibility of two different condensed phases on the basis of their observation of a sharp change in the apparent van't Hoff enthalpy of gelation at $22{ }^{\circ} \mathrm{C}$. Our studies show that $C_{\text {sat }}$ is always markedly greater than the minimal monomer concentration needed to produce measurable aggregation, $C_{\text {aur }}$, and therefore the overall solution to gel equilibrium cannot be treated as a single sharp polymerisation and phase condensation. $C_{\text {arr }}$ reflects polymerisation free energy, whereas $C_{\text {sat }}$, although also a monomer concentration, reflects the separation into isotropic and tactoidal phases.

The endothermic enthalpy for the entropy driven process of gelation has been measured by Ross et $a l^{12}{ }^{12}$ from the temperature dependences of $C_{\text {sat }}$ and birefringence observations, and by direct calorimetry. $\Delta H$ falls from $+4,000 \mathrm{cal} \mathrm{mol}^{-1}$ at $15^{\circ} \mathrm{C}$ to 2,200 at $25^{\circ} \mathrm{C}$ to zero at $37^{\circ} \mathrm{C}$. The large associated $\Delta C_{P}$ is $-197 \mathrm{cal}$ degree $\mathrm{mol}^{-1}$. Most of the enthalpy is associated with the polymerisation process, $\Delta H$ for alignment being in the neigbourhood of zero. These results are consistent with a polymerisation process dominated by hydrophobic interactions and alignment dependent on entropic forces as modelled by Minton ${ }^{5}$. The results of Magdoff-Fairchild et al. ${ }^{8}$ show similar $\Delta H$ values and also a $p \mathrm{H}$ dependence of $\Delta H$.

The three important physiological ligands of haemoglobin, oxygen, proton and 2,3 diphosphoglycerate (DPG) affect gelation. Thermodynamic studies (of $C_{\text {sat }}$ and $C_{\text {ags }}$ ) show that protons above $p H 6.8$ favour gelation and that an optimum for gelation exists near $p \mathrm{H} 6.8^{10,11,13}$. Protons also shorten the latent stage delay time ${ }^{14}$. In our studies DPG, and more so inositol hexaphosphate, also favour gel formation. On the other hand, under a single set of conditions resembling those in the erythrocyte, Swerdlow et $a l .{ }^{13}$ show no effect of DPG on gelation. Kinetic studies show that DPG shortens the delay time ${ }^{16}$. The oxygen Robin Briehl is in the Department of Physiology, Albert Einstein College of Medicine, New York.

\title{
Ice-Age palaeo-oceanography of the Mediterranean
}

\section{from N. J. Shackleton}

THE CLIMAP project had as its first major goal the reconstruction of global climate at the peak of the last glaciation about 18,000 years ago. One purpose of this was pure geological excitement, another was to use the resulting reconstruction as a boundary condition for numerical general circulation models of the atmosphere (for example, Gates Science 191, 1138; 1976). One of the most exciting moments in the CLIMAP project was the day we first compared the climate simulated by Gates' model for the ice age world, based on oceanographic data, with independent geological observations from the continents.

On the coarse grid-scale with which CLIMAP was forced to work the Mediterranean Sea looked rather insignificant, particularly considering the vast areas of Pacific we had to deal with, and it was at first treated rather summarily. However, it is an area of special interest to other workers, and Thiede has now presented a CLIMAP reconstruction of the Mediterranean alone (page 680 of this issue of Nature). The reconstruction is based on the Foraminifera, and its strength lies in the fact that it was possible to obtain a temperature calibration of the planktonic foraminiferal faunas of the Mediterranean utilising sediment samples from the North Atlantic and particularly from the Bay of Biscay. This is important because the glacial faunas clearly include some that do

equilibria of dilute haemolysates of haemoglobins $\mathrm{A}$ and $\mathrm{S}$ have long been known to be indistinguishable even though haemoglobin $S$ cells have a lower oxygen affinity than normal cells. Gill et $a l^{17}$ have now examined concentrated haemolysates and found that a concentration-dependent lowering of affinity exists for haemoglobin $\mathrm{S}$ at low saturations, associated with evidence of polymerisation by light scattering, but not at high saturations. The effect depends on the free energy change associated with polymerisation of the deoxygenated form.

In an extension of previous thermodynamic analyses, Minton ${ }^{18}$ and Ross and Minton ${ }^{19}$ have shown that the nonideality arising from the excluded volume of monomeric haemoglobin in solution makes a very large contribution to thermodynamic activity at the high haemoglobin concentrations not lie in the present range within the Mediterranean.

Glacial winter sea surface temperatures ranged from below $7^{\circ} \mathrm{C}$ on the entire Spanish and French coasts to more than $19^{\circ} \mathrm{C}$ on the Egyptian coast, while summer temperatures were below $13{ }^{\circ} \mathrm{C}$ along the Spanish and French coasts, rising to over $25^{\circ} \mathrm{C}$ in the South-East. Thus the Western Mediterranean appears to have been very considerably cooler than it is today, although in the South-Eastern corner conditions were not greatly different from today.

This reconstruction will be received with great interest by archaeologists who are exploring man's existence around the Mediterranean during the last glaciation. There is not nearly as much information as one might like preserved in some of the caves occupied around the Mediterranean for reconstructing the past environment, and a reconstruction of sea surface temperatures should provide a welcome input. One hopes that a synthesis will emerge which utilises the marine and terrestrial evidence together; at the least, it should be possible to calibrate Thiede's estimate of the seasonal temperature range by making oxygen isotopic measurements in some of the mollusc shells which prehistoric man so thoughtfully collected for our use.

N. J. Shackleton is at the SubDepartment of Quaternary Research, University of Cambridge.

required for gelation. The non-ideality as obtained from osmotic pressure data and the sedimentation equilibrium results of Williams ${ }^{20}$ and Briehl and Ewert $^{13}$ can be well accounted for under a model in which the haemoglobin molecule in solution is treated simply as a hard, impenetrable, quasispherical particle. Ross et al. demonstrate that this non-ideality suffices to explain the effects of some non-S haemoglobins on gelation in mixtures with haemoglobin $S$; no direct interactions of copolymerisation are required. The non-ideality thus explains the previously observed nonspecific facilitation of gelation of haemoglobin $S$ by the addition of haemoglobin $A$ or $F$ or ovalbumin ${ }^{21,22}$ and receives further confirmation from the demonstration that isolated $\alpha$ and $\beta$ subunits have the same effect on gelation of haemoglobins $\mathbf{S}$ as haemoglobin $\mathbf{A}^{23}$. 